\title{
Marco metodológico para la determinacion de la tasa de supervivencia empresarial en el sector industrial de la ciudad de Medellín en el periodo 2000-2010
}

\author{
Methodological framework for the determination of the rate of firms survival in Medellin \\ industrial sector between 2000-2010
}

\begin{abstract}
Resumen: En este trabajo se analiza la supervivencia de las empresas del sector industrial de Medellín creadas entre los años 2000 y 2010, en dónde la probabilidad de supervivencia se calcula con base en los tamaños de las empresas al momento de nacer, según el tipo de sociedad o forma jurídica y también por ramas o sectores de la industria. Los resultados derivados de la investigación que soporta el presente artículo evidencian que las pequeñas, medianas y grandes empresas disfrutan de mayores tasas relativas de supervivencia, mientras las microempresas encuentran más dificultades para continuar en actividad. De otro lado los resultados permiten demostrar que la innovación y la inversión mejoran la supervivencia de las nuevas empresas. Así mismo la fase inicial del ciclo de vida del sector favorece la entrada de empresas más que la salida, mientras que en aquellos que se encuentran en su fase madura 0 de declive, las salidas superan las entradas. Así mismo se encuentra que los aspectos regionales son determinantes en la supervivencia de las empresas.
\end{abstract}

Palabras clave: Creación de empresas; Innovación; Supervivencia empresarial; Pymes; Sector Industrial.

JEL: L200, L250, L260

\begin{abstract}
This paper analyzes the survival of the industrial firms of Medellin created between 2000 and 2010. The likelihood of survival is calculated based on company size at birth, according to the type of company or legal form as well as by industry sectors. Small, medium and large companies enjoy higher relative survival rates, while micro find it more difficult to continue in business. On the other hand, the results establish that the innovation and investment improve survival of new businesses. Likewise, the initial phase of the life cycle of the industry favors the entry of firms rather than itsoutput, while in those who are in their mature or declining phase outputs exceed inputs. Also, it is found that the regional aspects are crucial to the survival of businesses.
\end{abstract}

Key words: Firms creation; Innovation; Firms survival; SMEs; Industrial Sector.

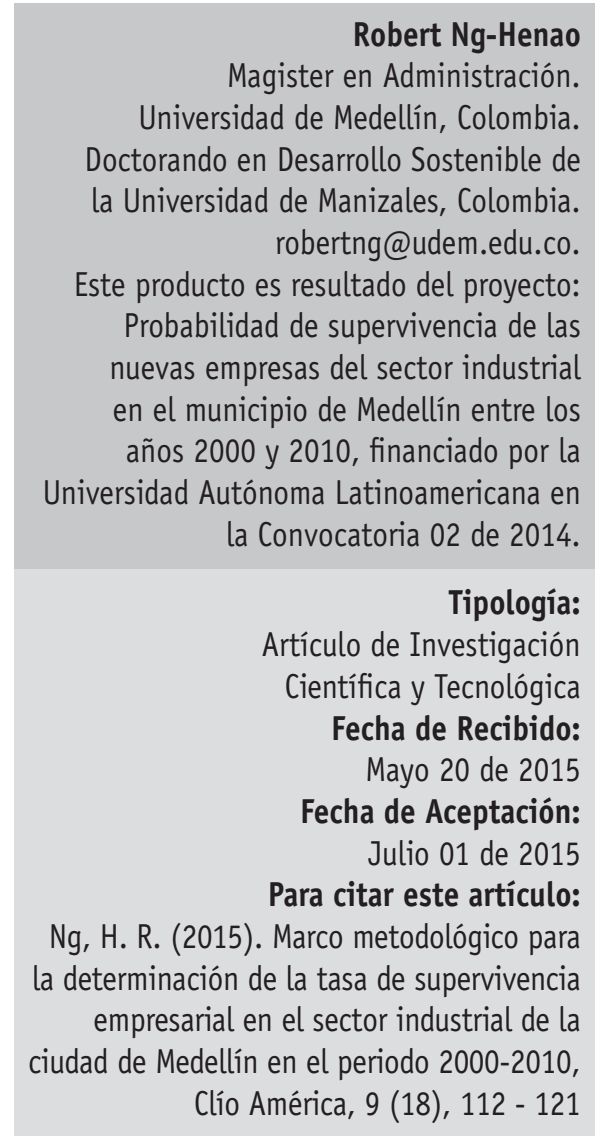




\section{Introducción}

El propósito del presente trabajo es el de investigar la probabilidad de supervivencia de las nuevas empresas creadas en el sector industrial de Medellín entre los años 2000 y 2010. Así mismo poner de manifiesto en qué medida se alcanza una consolidación de las empresas tras su nacimiento.

Se realizó un análisis de la literatura internacional sobre los principales determinantes de la supervivencia empresarial e igualmente sobre las más importantes recomendaciones y políticas que se han propuesto para favorecer la creación y consolidación de las empresas.

El estudio muestra cómo varían las dificultades de consolidación de las empresas en función de su tamaño inicial, según la naturaleza jurídica de las empresas al nacer, y de acuerdo también con el sector industrial al cual pertenecen.

Los resultados del trabajo ponen en evidencia que la entrada de empresas es mayor entre las microempresas, pero estas son también las que presentan las mayores tasas de mortalidad en los primeros años de vida ${ }^{1}$. Conforme a las Cámaras de Comercio, Industria y Navegación de España y Fundación INCYDE en el 2006, el riesgo de desaparición de una empresa disminuye conforme aumenta la antigüedad de la misma, y es menor en las empresas que nacen con un mayor tamaño (INCYDE, 2006, p. 37). De acuerdo con el tipo de sociedad, las personas naturales presentan las menores tasas de supervivencia. Por ramas industriales las menores tasas se de supervivencia se presentan en sectores tradicionales de la industria como alimentos, confecciones y textiles.

La creación de empresas resulta ser un proceso relativamente fácil de llevar a cabo, a juzgar por el número de organizaciones creadas en el período de

\footnotetext{
1. Uno de los esquemas más detallados acerca de "los primeros años de vida" de las empresas corresponde al trabajo desarrollado por Ana Guerrero de Rearte y Fernando Griaño (2004, p19), en dónde a través del trabajo “El proceso de creación de empresas, factores determinantes y diferencias espaciales" establecen que los primeros años de vida de cualquier estructura productiva obedece a lo que Gibbs y Ritchie (1982) contemplaban alrededor del proceso de gestación e infancia de las organizaciones, proceso que entre ambas transiciones puede tomar un periodo temporal cercano a los cinco años.
}

análisis, sin embargo, la supervivencia parece ser mucho más complicada (Maqueda, 2010, p. 57). Esto podría sugerir tal y como lo señalan Geroski (1995, p. 427) y Audretsch (1997, p.980), que más que barreras a la entrada de nuevas empresas, existen barreras a la supervivencia.

Gennero de Rearte y Liseras (2004, p.200) establecen un importante análisis de diferentes trabajos relacionados con la supervivencia de las empresas en sus primeros cinco años de vida, que son considerados una etapa crítica en el desarrollo y supervivencia en el mercado, debido a que buena parte de las nuevas firmas que nacen pequeñas, apenas llegas a superar los cinco primeros años de vida (Bennet, 1989). Por ello, es necesario reconocer y analizar los factores asociados a la supervivencia de las empresas en el mercado, así como el reconocimiento de los factores que contribuyen e inciden en un mejor desempeño durante dicho periodo de tiempo. Estos factores pueden ser catalogados en dos grandes categorías:

\section{Factores relacionados con el inicio de la organización:}

- Motivacionales: autoconfianza, toma de riesgos, creatividad, innovación, desplazamiento

- Recursos: ingreso individual, ingreso familiar, experiencia empresarial, nivel educativo

- Entorno: cultura empresarial

\section{Factores relacionados con el seguimiento:}

- Planeación y gestión: calidad y diseño de productos y servicios, publicidad y mercadeo, estimaciones de costos y presupuestos, formas de contratación de personal, mecanismos de financiación y apalancamiento

- Factores restrictivos y facilitadores: funcionamiento e integración del personal, asesoramiento profesional, entorno cercano, entorno o contexto macroeconómico

- La entrada y salida de empresas de los mercados ha constituido una de las formas más utilizadas por la economía industrial para explicar la evolución y adaptación de las actividades industriales al cambio. Este proceso se denomina Movilidad Empresarial. Es decir, la movilidad empresarial 
Marco metodológico para la determinacion de la tasa de supervivencia empresarial en el sector industrial de la ciudad de Medellín en el periodo 2000-2010

es el proceso dinámico de entradas y salidas de empresas que se producen en los mercados (Martí, 2000, p.13).

- Arribas (1993) afirma: "el modelo que más se utiliza en economía industrial para describir el funcionamiento de cualquier industria es el paradigma E-C-R (Estructura-Conducta-Resultados)" (p. 89), donde las variables que conforman la estructura del mercado: número de empresas, tamaño y distribución de las empresas, condiciones de entrada y salida, y tecnología empleada, entre otras. Estas características estructurales obligan a las empresas presentes en un sector particular a manifestar un comportamiento competitivo concreto expresado en los niveles de producción, precios, inversiones, publicidad, e investigación, desarrollo e innovación $(\mathrm{I}+\mathrm{D}+\mathrm{i})$, que repercute en los niveles de beneficio, eficiencia, calidad y nivel de empleo, entre otras variables.

- La escuela tradicional o de Harvard defiende una interpretación causal y unidireccional del paradigma E-C-R. Los resultados de cada industria dependen fundamentalmente de la estructura inicial de los mercados. La escuela de Chicago critica cualquier interpretación causal del paradigma E-C-R. La nueva economía industrial ${ }^{2}$ llega a una situación de síntesis en la que el paradigma E-C-R se sigue utilizando, pero sólo como una herramienta descriptiva, multidireccional, no causal de las industrias.

\section{La Creación de Empresas y el Desarrollo Económico}

De acuerdo con el economista austriaco Joseph Schumpeter, en su obra Capitalismo, socialismo y democracia, publicado en 1942, una de las grandes ventajas del capitalismo es el proceso de "destrucción creativa". Con ello, Schumpeter se refería al dinámico espíritu emprendedor siempre presente en las sociedades capitalistas, bajo el cual la búsqueda

\footnotetext{
2. La nueva economía industrial (NEI) adquiere mayor visibilidad con la publicación del libro de Tirole (1988), hace ya 20 años. Dos décadas en las que, como señala Ghemawat (1997, prefacio), el estudio económico de los mercados ha sido tenido muy poco en cuenta por los desarrollos en la disciplina de la Dirección Estratégica, sobre todo comparado con lo que la influencia de la economía Industrial en los primeros estadios de la disciplina a través de las obras de Porter (Salas, Fumás, 2007). Tirole organiza una buena parte de su libro alrededor de las variables competitivas sobre las que se concentra el comportamiento de las empresas en competencia.
}

de utilidades incentiva a los individuos y a las empresas a innovar ${ }^{3}$, a experimentar y a crear. Mediante este proceso desaparecen los productos obsoletos y también los métodos de producción utilizados por las empresas ineficientes, siendo reemplazados por nuevos y mejores diseños y por técnicas innovadoras puestas en práctica por empresas exitosas. A través del proceso de innovación que tiene lugar en las economías de mercado los nuevos productos destruyen viejas empresas y modelos de negocio.

Una economía eficiente es dinámica, con nuevas empresas innovadoras que reemplazan a empresas obsoletas 0 ineficientes. Si la cantidad de nuevas empresas innovadoras es insuficiente, el crecimiento a mediano y largo plazo será demasiado lento y habrá retraso económico relativo. De otro lado, sucede que si la creación de empresas tradicionales es muy elevada, el cierre de empresas será alto y el crecimiento y la supervivencia difícil (Callejón \& Ortún, 2009, p.172).

Cuando las estructuras productivas se envejecen sin impulsos creadores e innovadores que las dinamicen se tiene una muy limitada capacidad de adaptación ante condiciones económicas cambiantes (Graña \& Gennero de Rearte, 1999). Según Shane (2010) la creencia en el aporte de los emprendedores al empleo, la innovación y la productividad ha dado lugar a políticas de emprendimiento que buscan incrementar la cantidad de gente que emprende. Sin embargo, la contribución efectiva de la enorme mayoría de los emprendimientos es muy pobre, lo cual para el caso colombiano obedece en gran medida a que el emprendimiento obedece más a una necesidad que a una oportunidad, tal y como se perfila el proceso en otras economías tales como los Estados Unidos, Europa del Norte y Europa Oriental. Buena parte de los mismos no sobreviven más allá de la fase de gestación e infancia, la cual no llega a superar los 5 años, y los que sí lo logran suelen crear apenas su propio empleo o un par de puestos (p. 47).

\footnotetext{
3. Se da una innovación siempre que el proceso productivo experimenta una modificación cualitativa real. En el concepto de innovación se incluyen los siguientes casos: 1) introducción de un bien nuevo, es decir la introducción de un bien que no es familiar a los consumidores, o de una nueva calidad de un cierto bien; 2) introducción de un nuevo método de producción, esto es de un método que no haya sido probado aún en el sector productivo de que se trate; 3) apertura de un nuevo mercado; 4) conquista de una fuente de oferta de materias primas.
} 


\section{Teorías de la Movilidad Empresarial}

La movilidad empresarial radica en la capacidad que tienen las empresas de una misma industria y de un mismo mercado de ser más eficientes que las que se encuentran en sus mismas posibilidades.

\section{El Aprendizaje Pasivo de Jovanovic}

El aprendizaje pasivo se trata de una teoría de selección con información incompleta, según la cual las empresas aprenden de su eficiencia a medida que van operando en la industria. De acuerdo con Jovanovic (1982) las empresas podrían seguir una pauta de aprendizaje pasivo, en el sentido de que aprenden a evaluar su propia eficiencia y deciden entonces si crecen, no crecen, reducen tamaño o cierran, pero su nivel de eficacia no cambia en el tiempo (p.53).

\section{El Aprendizaje Activo de Ericson y Pakes}

Para Ericson y Pakes (1995) las empresas influyen en su capacidad de aprendizaje con sus decisiones de inversión; en consecuencia, el proceso de aprendizaje de la empresa adopta un carácter activo (p.75).

En tal sentido, la evidencia en Colombia establece que los modelos de aprendizaje activo en las empresas pueden cambiar sus características durante su permanencia en el mercado, según Martínez (2005) las causas de estos cambios pueden ser muy diversas (tecnológicas, organizativas o de cualquier otra índole) y, sus efectos, tanto positivos como negativos (p. 9).

La diferenciación tecnológica permite a las empresas agilizar el proceso de aprendizaje adoptando nuevas técnicas e introduciendo nuevos productos y procesos con el fin de ganar mayor poder de mercado y asegurar su supervivencia a lo largo del tiempo. En esencia, el aprendizaje activo se basa en la capacidad por parte de las empresas de ser más competitivas mediante la adopción de innovaciones tanto en producto como en la forma de producir u organizarse (Ortega-Argiles \& Moreno, 2005).

El paso de lo que se ha llamado "aprendizaje pasivo" a un tipo de "aprendizaje activo", pone de relieve en el proceso de crecimiento de la empresa las decisiones adoptadas por parte de la dirección que determinarán el comportamiento empresarial. Por lo tanto, el comportamiento empresarial viene dado por las decisiones que toman las empresas a lo largo de su vida, las cuales determinarán su eficiencia y a su vez su supervivencia.

\section{Metodología}

En este apartado se describen los conceptos empleados para realizar el análisis de la creación, cierre y supervivencia de empresas, como son las tasas de entrada y salida brutas, tasa de entrada neta, tasa de rotación, y las funciones de riesgo y de supervivencia.

\section{Fuentes de información}

La fuente de información para el cálculo de la tasa acumulada de supervivencia y la tasa de riesgo interanual es el Registro Público Mercantil de la Cámara de Comercio de Medellín, la cual proporcionó una base datos de las 19.671 empresas del sector industrial creadas entre los años 2000 y 2010.

Esta base suministra para cada empresa el número de identificación tributaria, la naturaleza jurídica, la razón social, dirección, fecha de matrícula, fecha de creación, fecha de disolución, duración en días, fecha de última renovación, activos iniciales, activos finales, clasificación CIIU a seis dígitos.

Con base en el valor de los activos y de acuerdo a la Ley 590 de 2000 Mipyme, se clasificaron las empresas definiendo su tamaño: microempresa, pequeña empresa, mediana y gran empresa; adicionalmente la clasificación CIIU se redujo a tres y dos dígitos.

Para las fechas de apertura y cierre, se adoptó como fecha de apertura la correspondiente a la de la matrícula en el registro mercantil y la fecha de cierre la correspondiente a la fecha de disolución. Es importante aclarar que a pesar de que algunas empresas registradas como inactivas no presentaban fecha de disolución, por parámetros definidos por la cámara de comercio, se decidió asignar para estos casos la fecha de última renovación del registro mercantil como fecha de cierre. 
Marco metodológico para la determinacion de la tasa de supervivencia empresarial en el sector industrial de la ciudad de Medellín en el periodo 2000-2010

Para efectos de análisis y presentación grafica de los datos, se consideró en una sola categoría a la mediana y gran empresa, debido a su baja representación dentro del universo de empresas de la muestra.

\section{Indicadores de la movilidad empresarial}

Los principales indicadores de la movilidad empresarial que se utilizaron para el desarrollo del trabajo se basan en la metodología acordada en el ámbito de la Unión Europea, que proporciona las directrices que permiten identificar poblaciones y generar indicadores relativos al stock, nacimiento, muerte y supervivencia de empresas, mediante una explotación estadística armonizada de los directorios de empresas gestionados en las oficinas de estadística. Estos indicadores fueron:

Tasa de entrada bruta: la tasa de entrada bruta de empresas muestra el porcentaje que representan las empresas nacidas en un cierto año respecto al número total de empresas existentes al inicio del año considerado (Eurostat, 2002, p.11).

Tasa de salida bruta: la tasa de mortalidad o tasa de salida bruta (TSB) es el porcentaje que representan las empresas que cierran en un cierto año en relación con las empresas existentes al inicio del año considerado (Eurostat, 2002, p.11).

Tasa de entrada neta: la tasa neta de entrada es la diferencia entre la tasa de entrada bruta y la tasa de salida bruta (Eurostat, 2002, p.12).

Tasa de rotación: se refiere a la suma de la tasa de entrada bruta y la tasa de salida bruta (Eurostat, 2002, p.12).

\section{Funciones de riesgo y de supervivencia}

El comportamiento de las empresas tras su nacimiento ha sido estudiado de forma sistemática por medio de funciones de riesgo y funciones de supervivencia. De acuerdo al trabajo de Ortega-Argiles y Moreno (2005, p. 12) el objetivo de estas funciones es conocer la relación entre la edad de la empresa y su tasa de mortalidad, lo que requiere estudiar la trayectoria de las empresas desde su nacimiento hasta su desaparición.
Para estudiar formalmente la relación existente entre la edad de una empresa y la probabilidad de salir del mercado se emplean las funciones de riesgo. De acuerdo a Segarra (2002, p. 17), y Segarra y Teruel (2006, p.24) la función de supervivencia en un cierto período muestra el porcentaje de empresas vivas transcurridas " $\mathrm{t}$ " períodos tras su aparición.

La función de riesgo muestra el porcentaje de empresas que cierra " $t$ " períodos después de su nacimiento. A su vez, la función de supervivencia recoge el porcentaje de empresas vivas " $t$ " períodos después del nacimiento (Spletzer, 1998, p.298).

\section{Estimador de la supervivencia de kaplan-meier}

Los modelos de duración, de supervivencia, sobrevivencia, o sobrevida, proporcionan técnicas para analizar el tiempo de seguimiento desde un instante inicial de observación hasta la ocurrencia de un evento de interés, tiempo de seguimiento que puede observarse completa o parcialmente (Kantis \& Juan, 2007, p. 59).

Para el caso del estudio en mención el origen coincide con la creación de la empresa (entrada) y el evento suele ser la muerte de la empresa (salida). El interés del análisis se centra en el tiempo que transcurre desde la entrada y el cierre.

En el presente estudio se desarrolla una aplicación empírica específica en el área de la movilidad empresarial. Concretamente se efectúa el cálculo de la supervivencia en la población (muestra de empresas) creadas entre los años 2000 y 2010.

El método de Kaplan-Meier (Kaplan \& Meier, 1958) se utiliza para analizar el tiempo de demora en ocurrir un fenómeno, aunque no se sabe si este ocurre o no.

La variable de interés en el análisis de duración es la longitud de tiempo que transcurre entre el momento en que un fenómeno empieza y el momento en que 0 bien el fenómeno finaliza o bien tiene lugar la medición, que puede ser un momento anterior al de finalización del fenómeno. Es decir en el momento en que se efectúa la medición el fenómeno puede no haber terminado. 
El concepto central de un modelo de supervivencia no es la probabilidad de que un cambio de estado ocurra, por ejemplo, la probabilidad que una empresa cierre, sino más bien la probabilidad condicional de que ocurra un cambio de estado, dado que tenía en el tiempo anterior otro estado, es decir, que una empresa cierre dado que en el periodo anterior no lo había hecho (Ayala \& Borges, 2007, p.32).

Siguiendo las explicaciones de Mata (1995, p.470) y de Harris y Hassaszadeh $(2002$, p.310) la tasa de supervivencia (survival rate) de una empresa individual o de un grupo de empresas en el ejercicio " $t$ " muestra el número de empresas que continúan activas en " $\mathrm{t}$ " con relación al número inicial de empresas. Es decir:

\section{$S(t)=$ Empresas Activas en " $t$ "/ Número inicial de empresas}

La probabilidad de que una empresa individual (o un grupo de empresas) finalice su actividad durante un determinado ejercicio " $t$ " estará en función del riesgo que recae sobre la empresa (hazard rate). La tasa de riesgo de las empresas activas en "t-1" que lograron sobrevivir en " $t$ " se expresa así:

$$
h(t)=1-\left[\frac{S(t)}{S(t-1)}\right]
$$

Esta expresión, de acuerdo a los aportes de Harris y Hassaszadeh (2002) establece la probabilidad de que una empresa de " $\mathrm{t}-1$ " años salga del mercado durante el período " $\mathrm{t}$ ". Si el tiempo adopta una dimensión discreta, la tasa de riesgo $h(t)$ refleja la probabilidad de que una empresa que ha sobrevivido hasta el ejercicio " $t$ " salga del mercado en el período " $\mathrm{t}+\Delta \mathrm{t}$ " tal como se puede observar en la siguiente expresión (p.315).

$$
h(t)=\lim _{\Delta t \rightarrow o}\left(\frac{P\left(t \leq T \leq t+\frac{\Delta t}{T} \geq t\right.}{\Delta t}\right)^{1}=\mathrm{f}(\mathrm{t}) / \mathrm{S}(\mathrm{t})
$$

donde $t=1,2, \ldots T$, es el tiempo discreto; $f(t)=d F(t) /$ $d t$ es la función de densidad correspondiente a la distribución de las salidas respecto al número inicial de empresas del grupo; $F(t)=\operatorname{Pr}(T<t)$ es la probabilidad de que las empresas del grupo alcancen un período vital "T" inferior a " $t$ "; $y$, por último, $S(t)=1-F(t)$ es la función de supervivencia (Dolton \& Van der Klauw, 1995, p.439).

La función de supervivencia por el método de Kaplan-Meier, se calcula mediante una fórmula recursiva de probabilidades condicionales.

$$
S\left(t_{\mathrm{j}}\right)=\frac{n_{\mathrm{j}}-d_{\mathrm{j}}}{n_{\mathrm{j}}} * S\left(t_{\mathrm{j}-1}\right)
$$

De acuerdo al modelo propuesto por Kaplan y Meier (1958) para un año determinado en un período dado de tiempo la supervivencia empresarial se calcula como la supervivencia acumulada en el año inmediatamente anterior multiplicada por la supervivencia en ese año. En el denominador se tiene el número de empresas activas, $n_{i}$, expuestas al riesgo, y en el numerador a ese valor se resta el número de empresas, $d_{j^{\prime}}$ que registran cierre en ese año; donde:

$n_{j}=$ Número de empresas activas en $t_{j}$ Número de empresas en riesgo en $t_{j}$

$d_{j}=$ Número de empresas que cierran en $t_{j}$

$\lambda j=d_{j} / n_{i}$ se conoce como el estimador no paramétrico de Kaplan-Meier o también el estimador del producto límite ( $p .462)$

Dolton y Van der Klauw (1995, p.440) evidencian lo propuesto por Kaplan y Meier (1958, p.460) en función a que este estimador incorpora información de todas las observaciones disponibles, sean o no censuradas. Además, es una función que depende solo de los valores de la muestra y permite describir la relación existente entre la función de riesgo y el tiempo de supervivencia hasta que ocurre el evento de interés.

$$
S(t j)=1-\lambda j * S(t j-1)
$$

Una forma alternativa de escribir la función de supervivencia es entonces: 


$$
S(t j) \prod_{i=0}^{j-1}(1-\lambda j)
$$

Por lo tanto, para ilustrar cómo se pretenden construir las tasas acumuladas de supervivencia y las tasas interanuales de riesgo, a continuación se presentan dos cuadros resumen para la cohorte correspondiente al año de inicio del análisis, el año 2000. Así luego de transcurrido un año, el riesgo de que una empresa salga en el próximo periodo, dado que se mantuvo hasta el año inmediatamente anterior es de $12.6 \%$, calculado como sigue:

$n_{j}=1768$ (Empresas activas en el año 2001) $d_{j}=223$ (Empresas que cerraron en el año 2001)

$\lambda j=d_{j} / n \Longrightarrow \lambda j=223 / 1768 \Longrightarrow \lambda j=0.1261$

En tal sentido, la tasa de riesgo, considerada como el estimador paramétrico de Kaplan-Meir o estimador del producto límite para los años siguientes que comprende el estudio, se pueden analizar de acuerdo a la información contemplada en la tabla número 1, que se enseña a continuación:

Tabla 1.

Tasa de riesgo (estimador no paramétrico de Kaplan-Meier) para las empresas del sector industrial de la ciudad de Medellín entre los años 2000 y 2010.

\begin{tabular}{|c|c|c|c|c|}
\hline Año & $\begin{array}{c}\text { Empresas activas al año } \\
\text { de inicio }\end{array}$ & $\begin{array}{c}\text { Bajas registradas duran- } \\
\text { te el año }\end{array}$ & Tasa de riesgo $\lambda=\mathrm{d} / \mathbf{n}$ & Tasa de riesgo \% \\
\hline 2000 & 1841 & 73 & 0.0397 & 4.0 \\
\hline 2001 & 1768 & 223 & 0.1261 & 12.6 \\
\hline 2002 & 1545 & 149 & 0.0964 & 9.6 \\
\hline 2003 & 1396 & 69 & 0.0494 & 4.9 \\
\hline 2004 & 1327 & 45 & 0.0339 & 3.4 \\
\hline 2005 & 1282 & 69 & 0.0538 & 5.4 \\
\hline 2006 & 1213 & 31 & 0.0256 & 2.6 \\
\hline 2007 & 1182 & 42 & 0.0355 & 3.6 \\
\hline 2009 & 1140 & 31 & 0.0272 & 2.7 \\
\hline 2010 & 1109 & 26 & 0.0234 & 2.3 \\
\hline
\end{tabular}

Fuente: Elaboración propia. 
El cálculo de las tasas acumuladas de supervivencia para la misma cohorte se presenta a continuación. Por ejemplo la tasa de supervivencia dos años después de creada la empresa se calcula en la forma siguiente:

$$
S(t j)=1-\lambda j * S(t j-1)
$$

Para el año 2000 la expresión es igual a 0,9636. Luego tasa acumulada de supervivencia en el año 2000 es igual a, es decir 0,9636. Para el año 2001 el valor de es igual a 0.8739 , por lo tanto si multiplicamos este valor por la tasa de supervivencia acumulada del año anterior podremos observar que la tasa de supervivencia para el año 2001 será igual a 0.8392, es decir, una tasa acumulada de supervivencia para las empresas del sector industrial de la ciudad del Medellín del 83.92\%. De acuerdo con la metodología planteada, podemos observar los resultados entre los años 2000 y 2010 en la tabla número 2 que se expone a continuación:

Tabla 2.

Tasa de Supervivencia para las empresas del sector industrial de la ciudad de Medellin entre los años 2000 y 2010.

\begin{tabular}{|c|c|c|}
\hline Año & Parámetro 1- $\lambda$ & Tasa de Supervivencia \\
\hline 2000 & 0.9603 & 0.9603 \\
\hline 2001 & 0.8739 & 0.8392 \\
\hline 2002 & 0.9036 & 0.7583 \\
\hline 2003 & 0.9506 & 0.7208 \\
\hline 2004 & 0.9661 & 0.6964 \\
\hline 2005 & 0.9462 & 0.6589 \\
\hline 2006 & 0.9744 & 0.6420 \\
\hline 2007 & 0.9645 & 0.6192 \\
\hline 2008 & 0.9728 & 0.6024 \\
\hline 2009 & 0.9766 & 0.5883 \\
\hline 2010 & 0.9548 & 0.5617 \\
\hline
\end{tabular}

Fuente: Elaboración propia.

\section{Resultados}

Numerosos trabajos han estudiado el impacto de la creación de empresas sobre el crecimiento económico. El análisis de la creación de empresas en general se encuentra en fase de revisión después de comprobar que la enorme heterogeneidad de las empresas que recién se crean dificulta la interpretación de los efectos económicos agregados. En una nueva línea de trabajo se busca en cambio identificar las características específicas que les permite a muchas empresas crecer, generar empleo cualificado, abrir nuevos nichos de mercado o ganar mercado internacional. Ciertamente solo una parte de las empresas que se crean contribuye en grado relevante a tales metas.

Por ello es necesario centrar esfuerzos fundamentalmente en emprendedores con verdadero espíritu empresarial, pues resulta complicado orientar hacia la actividad empresarial a quien no muestra interés o vocación por la misma, o lo hace obligado por la necesidad. Los responsables de la política, deberían motivar la realización de diversos análisis sobre las posibilidades de éxito de los proyectos empresariales, ya que si bien es cierto que el autoempleo es una alternativa de salida profesional, solo en aquellos casos donde confluyan determinadas aptitudes y actitudes de la persona puede surgir un proyecto empresarial con perspectivas de consolidación, sin importar el sector de la economía hacia el que se enfoque.

No obstante, en períodos de estancamiento económico y crecimiento del desempleo los gobiernos locales pueden ofrecer apoyos muy valiosos a través de programas de formación, de asesoramiento, de oferta de espacios equipados, y microcréditos, entre otros, para la creación de empresas. Aunque en verdad muy pocas de estas empresas logran luego prosperar, crecer y generar empleo adicional al del propietario.

Es necesario la concertación de objetivos claros y políticas adecuadas en materia de los proceso de 
Marco metodológico para la determinacion de la tasa de supervivencia empresarial en el sector industrial de la ciudad de Medellín en el periodo 2000-2010

emprendimiento, esto con el fin de disponer de los instrumentos y herramientas de política necesarias y apropiadas; de igual forma se debe contar con la suficiente claridad alrededor del perfil y orientación de los emprendimientos, para poder establecer estrategias selectivas de captación, coherentes con los objetivos y perfiles, en tal sentido es importante señalar que es igual de importante comprender el sector de la economía en el que se planean desarrollar los emprendimientos, en razón a que hay sectores tales como el sector de los servicios, en donde los costos de producción y las inversiones iniciales en tecnología y capital humano implican un mayor esfuerzo por parte de todos los agentes económicos involucrados.

Es igualmente preciso diferenciar entre los casos de autoempleo y los proyectos empresariales de más alcance. Se trata de categorías diferentes que deben ser abordadas con programas específicos. Esta focalización es fundamental para la promoción de nuevas empresas con posibilidad de crecer e impactar en variables como la innovación, el avance de la estructura productiva y la internacionalización.

Los riesgos derivados de la supervivencia empresarial también se ven sometidos al desempeño de las principales variables que rodean el devenir del contexto macroeconómico del país. Para el caso colombiano es importante señalar la existencia de una correlación positiva entre la fase del ciclo económico por la que se está atravesando y el riesgo de supervivencia de las nuevas empresas, las cuales se hacen mucho más propensas al fracaso, exigiendo por parte del staff directivo de las mismas un marco mucho más estratégico de reacción en el proceso de toma de decisiones, al mismo tiempo que el desarrollo de una mayor sinergia con los demás agentes económicos que le rodean, en especial el estado y el sector de la educación, la ciencia, la tecnología y la innovación.

\section{Conclusión}

Con respecto a la aplicación del marco metodológico propuesto para el cálculo de la supervivencia empresarial de las empresas del sector industrial de la ciudad de Medellín entre los años 2000 y 2010, se pueden concluir los siguientes aspectos:
- El riesgo de desaparición de una empresa disminuye conforme aumenta la antigüedad de la misma, y es menor en las empresas que nacen con un mayor tamaño

- Las dificultades de permanencia de las empresas están asociadas en un alto porcentaje a la incidencia de la fase del ciclo económico por la que se esté atravesando y a las proyecciones de crecimiento económico hacia el corto y mediano plazo

- La creación de empresas es mayor entre las de menor tamaño, pero estas empresas son también las que presentan las mayores tasas de mortalidad en los primeros años de vida

- La supervivencia de las nuevas empresas se encuentra positivamente relacionada con su tamaño al nacer. A medida que aumenta el tamaño empresarial la probabilidad de supervivencia de la empresa es mayor. Es un hecho estilizado documentado en la literatura sobre movilidad empresarial, existiendo muchos trabajos que llegan a las mismas conclusiones

- De otro lado, se ha encontrado que la innovación y la inversión mejoran la supervivencia de las nuevas empresas. Así mismo la fase inicial del ciclo de vida del sector favorece la entrada de empresas más que la salida, mientras que en aquellos que se encuentran en su fase madura 0 de declive las salidas superan las entradas. Así mismo se encuentra que los aspectos regionales son determinantes en la supervivencia de las empresas.

- A partir del análisis de las funciones de riesgo y supervivencia obtenidas mediante la aplicación del modelo Kaplan-Meier se concluyó que tras el primer año de vida de la cohorte del año 2000, cerraron el $12,6 \%$ de las empresas, dos años después el 9,6\%, y nueve años después el 2,3\% de las empresas

\section{Referencias Bibliograficas}

Arribas, E. H. (1993). Contribuciones de la organización industrial a la división estratégica de la empresa. Proyecto social: Revista de relaciones laborales, (1), 85-108.

Audretsch, D., Santarelli, E., \& Vivarelli, M. (1997). Start-up size and industrial dynamics: some evidence from italian manufacturing. International Journal of Industrial Organization. Número 17, 965-983. 
Ayala, M. B., \& Borges, R. E. (2007). Análisis de supervivencia aplicado a la Banca Comercial Venezolana 1996-2004. Mérida, Venezuela. Instituto de Investigaciones Económicas y Sociales, Universidad de Los Andes

Cámaras de Comercio Industria y Navegación de España y Fundación INCYDE (2006): “La creación de empresas en España. Análisis por sectores y Regiones". Ed. Cámaras de comercio; Industria y Navegación de España. Madrid, pág. 40.

Callejón, M. 0., \& Ortún, V. (2009). La caja negra de la dinámica empresarial. Investigaciones Regionales. Número 15. 167-189.

Dolton, P. J., and W. Van Der Klauw (1995): “Leaving Teaching in the UK: A Duration Analysis," Economic Journal, 105, 431-444.

Ericson, R. \& Pakes, A. (1995): “Markov-Perfect Industry Dynamics: a Framework for Empirical Work". Review of Economic Studies, Número 62. 53-82.

Eurostat (2002): "Structural Indicators". Documents Eurostat: Definitions - Data sources - Data availability, Número D2. 1-29.

Geroski, P. A. (1995): "What do we know about entry". International Journal of Industrial Organization. London Business Scool, Sussex Place, Regent"s park, Número 13. 421-440.

Graña, F., \& Gennero de Rearte, A. (1999). Patrones de nacimiento, mortalidad y supervivencia de firmas industriales en un período de transición: un análisis de caso, 19891994. Mar del Plata, Argentina. Universidad Nacional de la Plata.

Harris, R. I. D., and P. Hassaszadeh (2002): "The Impact of Ownership Changes and Age Effects on Plant Exits in UK Manufacturing, 1974-1995.," Economics Letters, 75, 309-317.
Jovanovic, B. (1982): "Selection and Evolution of Industry". Econometrica. Número 50. 649-670.

Kaplan, E. L. \& Meier, P. (1958), “Nonparametric Estimation from incomplete Observations", Journal of the American Statistical Association. Número 53, 457-481.

Martí, F. P. (2000). La Movilidad Empresarial en la industria Española. Alcalá, España. Editorial Alcalá.

Martínez, A. (2005): “Creación y Consolidación de Empresas en el Área Metropolitana de Cali 1996-2002", Revista del Observatorio Económico del Valle del Cauca, № 6.

Mata, J., P. Portugal, and P. Guimaraes (1995): "The Survival of New Plants: Start-up Conditions and PostEntry Evolution," International Journal of Industrial Organization, 13, 459-481

Maqueda, F.J. (2010). Marketing, innovación y nuevos negocios. Libros profesionales de empresa. ESIC Editorial.

Ortega-Argiles, R., \& Moreno, R. (2005). Estrategias Competitivas y Supervivencia empresarial. Barcelona, España. Universidad de Barcelona.

Segarra, A. (2002). Supervivencia empresarial, ciclo de vida y régimen tecnológico. España. Facultad de Ciencias Económicas y empresariales. Universidad de Rovira i Virgili.

Segarra, A. y., \& Teruel, M. (2006). Creación y supervivencia de las nuevas empresas en las manufacturas y los servicios. España. Facultad de Ciencias Económicas y empresariales. Universidad de Rovira i Virgili.

Shane, S. (2010). The Illusions of Entrepreneurship. Estados Unidos. Yale University.

Spletzer, J (1998), "The Contribution of Establishment Births and Deaths to Employment Growth", Bureau of Labor Statistics WP-310. 